\title{
Performance of Thermally Excited Resonators
}

\author{
T S J LAMMERINK, M ELWENSPOEK, R H VAN OUWERKERK, S BOUWSTRA and J H J FLUITMAN \\ Sensors and Actuators Research Unit, Unwersity of Twente, PO Box 217, 7500 AE Enschede (The Netherlands)
}

\begin{abstract}
A study of electrothermal excitation of micromachined silicon beams is reported The temperature distribution is calculated as a function of the position of the transducer, resulting in stress in the structure which reduces the resonance frequency Test samples are realized and measurements of resonance frequency, vibration shape and vibration amplitude are carried out There is a satisfactory agreement between theory and experıment at small thermal stresses Near the buckling load we find distinct deviations from theory which are ascribed to mechanical imperfections of the beams
\end{abstract}

\section{Introduction}

Recently, much interest has been shown in sensors based on integrated resonators with a measurand-dependent resonance frequency [1-7] Such a resonator has to be excited and the resulting vibration detected in order to measure the resonance frequency Electro- or optothermal excitation of the resonator has advantages from a technological point of view Wilfinger et al [8] were the first to use electrothermal excitation for a resonistor Static heat elevation of the resonating structure is inherent in thermomechanical excitation, resulting in mechanical stresses which influence the resonance frequency and vibration amplitude Therefore it is interesting to design a resonator with its excitator constructed so that there is a minımum temperature elevation at a maximum vibration amplitude In this paper we focus our attention on a clamped-clamped beam resonator with a thin-film resistor as electrothermal excitator We calculate the influence of the position of the thin-film resistor on the average temperature elevation and the resonance frequency shift as well as its influence on the vibration amplitude of the beam and compare the results with measurements on realized test samples

\section{Mechanical Model}

The resonator $(\mathrm{F} 1 \mathrm{~g} \quad \mathrm{l}(\mathrm{a})$ ) is regarded as a homogeneous, prismatic clamped-clamped beam forced into vibration by a distributed driving moment $M_{\mathrm{d}}(x)$ Without damping its vibration is described by the partial differential equation

$$
\begin{gathered}
E I \frac{\partial^{4} W(x, t)}{\partial x^{4}}-\sigma A \frac{\partial^{2} W(x, t)}{\partial x^{2}}+\rho A \frac{\partial^{2} W(x, t)}{\partial t^{2}} \\
=\frac{\partial^{2} M_{\mathrm{d}}(x, t)}{\partial x^{2}}
\end{gathered}
$$

with $W(x, t)$ the displacement function, $E I$ the bending stiffness and $A$ the cross section of the beam, $\sigma$ the axial stress and $\rho$ the specific mass of the beam material Solution of eqn (1) (with the help of Laplace transformation) for $\sigma=0$ and a harmonic place-dependent driving moment $M_{\mathrm{d}}(x)$ (see Fig 1(b)) results in an analytical expression

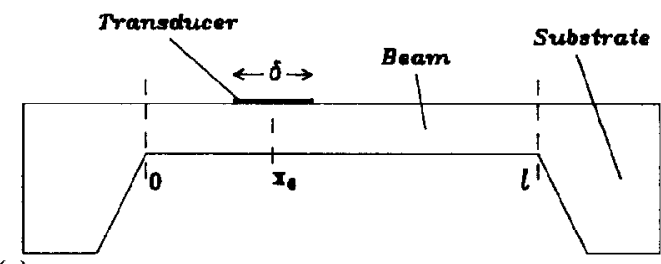

(a)
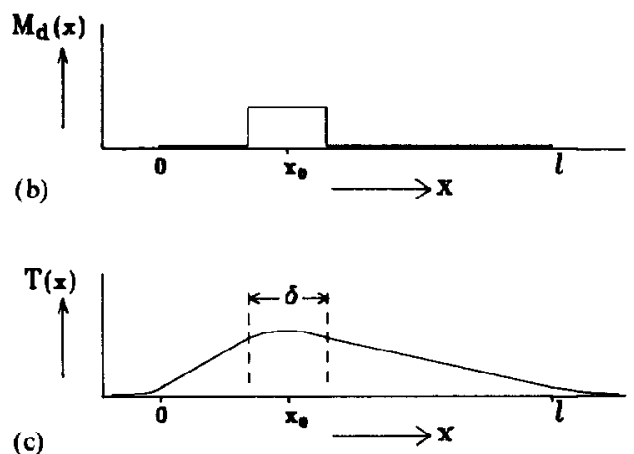

Fig 1 (a) Simplified geometry of a beam, (b) driving moment $M_{\mathrm{d}}(x)$, (c) temperature function with uniform heat generation in resistor and finite conductivity of the substrate

(C) Elsevier Sequoia/Printed in The Netherlands 
for the frequency-dependent vibration shape $W(x, t)$ The model results in line symmetrical (even modes) or point symmetrical (odd modes) shape functions $W(x)$ for the beam resonance frequencies (see Fig 4) It is seen from eqn (1) that the vibration amplitude depends on the shape of the moment function $M_{\mathrm{d}}(x)$, hence on $x_{\mathrm{o}} / l$ and $\delta / l$ in Fig 1(b) The expected vibration amplitude at the first resonance frequency as a function of $x_{\mathrm{o}} / l$ (with $\delta / l=02$ and $M_{\mathrm{d}}=$ constant) is given (see Fig 8) The eigenvalues of eqn (1) result in the stress-dependent resonance frequencies of the beam The first-order resonance is approximated with

$f_{\text {res }} \cong f_{0}\left(1+\sigma / \sigma_{\mathrm{cr}}\right)^{1 / 2}$

where $f_{\mathrm{o}}$ is the resonance frequency with no stress in the beam $(\sigma=0), f_{\mathrm{o}}=1028(E / \rho)^{1 / 2} h / l^{2}$, with $E$ the effective Young's modulus, $\rho$ the specific mass and $h$ and $l$ the thickness and the length of the beam, respectively The critical Euler buckling load $\sigma_{\mathrm{cr}}=4 \pi^{2} E I / A l^{2}[9]$

\section{Heat Generation and Thermal Model}

We now need to calculate the stress from the temperature distribution The film resistor on top of the beam (Fig 1(a)) is driven by a harmonic a c voltage $U_{\text {ac }} \cos \omega t$ superimposed on a d c voltage $U_{\mathrm{dc}}$ The electrothermally generated heat is given by

$$
\begin{aligned}
P(t)= & \frac{\left(U_{\mathrm{dc}}{ }^{2}+05 U_{\mathrm{ac}}{ }^{2}\right)}{R}+\frac{2 U_{\mathrm{dc}} U_{\mathrm{ac}} \cos \omega t}{R} \\
& +\frac{05 U_{\mathrm{ac}}{ }^{2} \cos 2 \omega t}{R}
\end{aligned}
$$

with $R$ the electrical resistance of the resistor The generated heat consists of one static component $P_{\text {stat }}=\left(U_{\mathrm{dc}}{ }^{2}+05 U_{\mathrm{ac}}{ }^{2}\right) / R$ and two dynamic components of amplitude $P_{\mathrm{dyn} \mathrm{I}}=2 U_{\mathrm{dc}} U_{\mathrm{ac}} / R$ and $P_{\text {dyn } 2}=05 U_{\mathrm{ac}}{ }^{2} / R$ respectively The static component results in a static temperature distribution With a uniform heat generation over the resistor and assuming that all the heat flows through the beam (by means of conduction) towards the clamped edges which are assumed to be ideal heat sinks, the temperature distribution is parabolic under the resistor and linear outside, as shown in Fig 1(c) For the heat generation concentrated at the centre $x_{0}$ of the resistor, the average temperature elevation $\Delta T_{\text {av }}$ integrated over the beam depends on $x_{0} / l$ and is given by

$\Delta T_{\mathrm{av}}=P_{\mathrm{stat}} \frac{l\left[x_{\mathrm{o}} / l-\left(x_{\mathrm{o}} / l\right)^{2}\right]}{2 b h \lambda}$

with $b, h$ and $l$ the width, the thickness and the length of the beam, respectively and $\lambda$ the heat conductivity of the beam material (assumed here to be independent of the temperature elevation) With $\lambda=157 \mathrm{~W} /\left(\mathrm{m}^{\circ} \mathrm{C}\right)$ for silicon, beam dimensions $b=1 \mathrm{~mm}, h=30 \mu \mathrm{m}, l=10 \mathrm{~mm}$ and position $x_{\mathrm{o}} / l=05$, we find that $\Delta T_{\mathrm{av}} / P_{\text {stat }}=$ $265^{\circ} \mathrm{C} / \mathrm{W}$ With a distributed heat generation over the resistor and $\delta / l=02$, we find that $\Delta T_{\text {av }} /$ $P_{\text {stat }}=261{ }^{\circ} \mathrm{C} / \mathrm{W}$ (see $F_{1} g$ 6) Two-dimensional finite-element numerical analysis with the heat sink at the substrate bottom shows a temperature distribution which deviates from the analytical model at the resistor location and the clamped edges of the beam At both locations a temperature difference between the top and the bottom surfaces of the beam results in a local static mechanical moment Due to the finte thermal conductivity of the substrate, the numerical analysis predicts a slightly higher $(2 \%)$ value for average temperature elevation $\Delta T_{\mathrm{av}}$ If we assume an extra thermal impedance $R_{3}$ between the beam ends and the surroundings, the average temperature $\Delta T_{\mathrm{av}}$ relative to the surroundings significantly increases (see Fig 6, where the extra impedance is $\left.02 R_{\mathrm{b}}=02 l / b h \lambda\right)$

The average temperature elevation $\Delta T_{\mathrm{av}}$ of the beam induces a compressive axial stress $\sigma=-E \alpha$ $\Delta T_{\text {av }}$, with $E$ the Young's modulus and $\alpha$ the coefficient of thermal expansion The relation between the first-order resonance frequency $f_{\text {res }}$ and $P_{\text {stat }}$ (see Fig 5) can be found from the relation between the stress and the average temperature elevation $\Delta T_{\mathrm{av}}$, the critical Euler load $\sigma_{\mathrm{cr}}$ and eqn

(2) The critical static power generation at which the beam will buckle is given by

$P_{\text {stater }}=\frac{2 \pi^{2}}{3} \frac{\lambda b}{\alpha\left[x_{\mathrm{o}} / l-\left(x_{\mathrm{o}} / l\right)^{2}\right]}\left(\frac{h}{l}\right)^{3}$

with $x_{\mathrm{o}} / l$ the relative transducer position For a beam with $b=1 \mathrm{~mm}, h=30 \mu \mathrm{m}, l=10 \mathrm{~mm}$, $x_{0} / l=05, \alpha=25 \times 10^{-6} /{ }^{\circ} \mathrm{C}$ and $\lambda=157 \mathrm{~W} /$ $\left(\mathrm{m}^{\circ} \mathrm{C}\right)$ the beam will buckle at $P_{\text {statcr }}=446 \mathrm{~mW}$ with $\Delta T_{\text {avcr }}=118^{\circ} \mathrm{C}$

The beam vibration is excited with the additional harmonic temperature distribution induced by the dynamic power with amplitude $P_{\text {dynl }}$ The thermal gradients (and so a thermal moment) in the thickness direction of the beam result in a proportional mechanical moment $M_{\mathrm{d}}(x, t)$, which excites the beam into vibration [10]

\section{Realization of Test Samples}

Figure 2 shows a test sample with a beam width of $1 \mathrm{~mm}$ and a length of $10 \mathrm{~mm}$ The thinfilm resistor covers the width of the beam by $2 \mathrm{~mm}$ The centre positions $x_{0}$ are at $0,1,2,3,4$ 


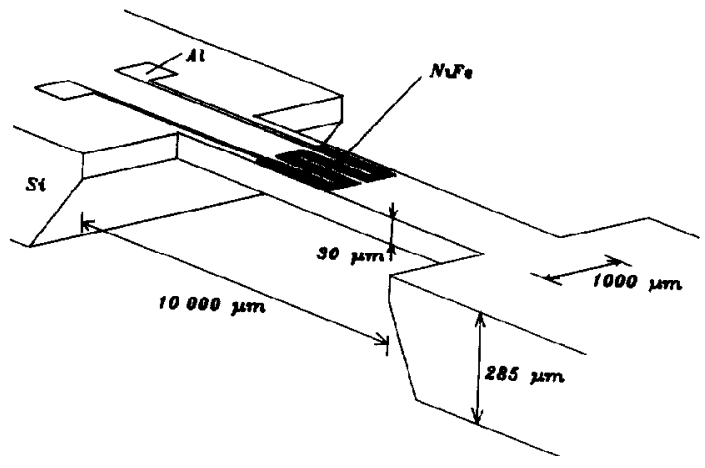

Fig 2 Lay-out of the clamped-clamped beams with a $\mathrm{N}_{1}-\mathrm{Fe}$ film as electrothermal transducer and alumınium metallization

or $5 \mathrm{~mm}$ from the beam end with a final beam thickness of $30 \mu \mathrm{m}$

The starting material is a 2 in (100) phosphorus-doped n-type silicon wafer polished on one side First alignment markings on both sides of the wafer are realized Then $\mathrm{S}_{1} \mathrm{O}_{2}$ layer of $1 \mu \mathrm{m}$ $\left(3 \mathrm{~h}, 1150^{\circ} \mathrm{C}\right.$, wet $\left.\mathrm{N}_{2}\right)$ is grown and removed from the top side of the wafer The resistor is realized from an evaporated $\mathrm{Al}$ film $(03 \mu \mathrm{m})$ and a $\mathrm{N} 1-\mathrm{Fe}$ layer $(20-80 \%$, RF sputtered, $250 \mathrm{~W}$, $6 \mathrm{~min}, 50 \mathrm{~nm}$ ) Both layers are patterned by wet chemical etching The beams are dry-etched from a membrane (anisotropic etch $\mathrm{KOH}$ in $\mathrm{H}_{2} \mathrm{O}$, $\left.50 \mathrm{~g} / 100 \mathrm{ml}, 75^{\circ} \mathrm{C}\right)$ in a $\mathrm{SF}_{6}$ plasma $(12 \mu \mathrm{m} / \mathrm{mun})$ with a positive resist etch mask The back $\mathrm{S}_{1} \mathrm{O}_{2}$ layer is removed to reduce the axial stress in the beams

\section{Experimental}

We used a Michelson interferometer and a Mach-Zehnder heterodyne interferometer for measuring the beam vibration The Michelson interferometer 15 based on a $5 \mathrm{~mW} \mathrm{He}-\mathrm{Ne}$ gas laser with a beam width of $06 \mathrm{~mm}$ For constant sensitivity the interferometer is kept in quadrature with a low frequency feedback loop and a piezocontrolled reference mirror A gain/phase analyser (HP 4194A) is used to determine the resonance frequencies and their quality factors Figure 3 shows a typical result obtained with this set-up The Mach-Zehnder heterodyne interferometer is based on a $10 \mathrm{~mW} \mathrm{He}-\mathrm{Ne}$ gas laser and has a measuring spot of $40 \mu \mathrm{m}$ diameter in combination with a computer-controlled device translation table Resonance frequency shifts of $30 \%$ are observed due to mounting the wafer on the device

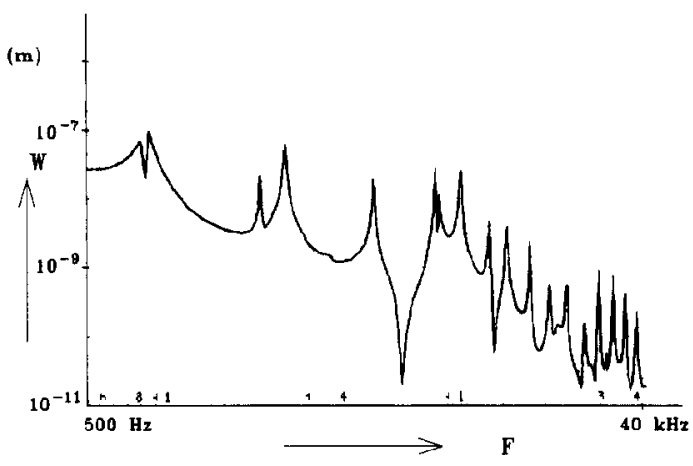

Fig 3 Vibration amplitude of a beam as a function of the frequency measured with a Michelson interferometer The dip at the first-order resonance $f=800 \mathrm{~Hz}$ is due to the strong non-linearity of the interferometer The second resonance at $f=2 \mathrm{kHz}$ does not belong to the collection of transversal resonance modes

holder and to forces induced by the probe pens used for contacting the resistor

\section{Results and Discussion}

Figure 4 shows both the measured and the predicted beam vibration shapes for the first- and the second-order resonances The measured shapes are qualitatively similar to the predicted ones The differences may be due to the temperature distribution, or inhomogeneous mechanical properties and are the subject of current investigation

The resonance frequencies of the beams as a function of the static power $P_{\text {stat }}$ are given in Fig 5 The frequency values are normalized at the resonance frequency for the stress-free beam With a thickness of $h=30 \mu \mathrm{m}$ we expect $f_{\mathrm{o}}=2633 \mathrm{kHz}$ The measured $f_{0}$ values for the different beams vary from $f_{\mathrm{o}}=215 \mathrm{kHz}$ to

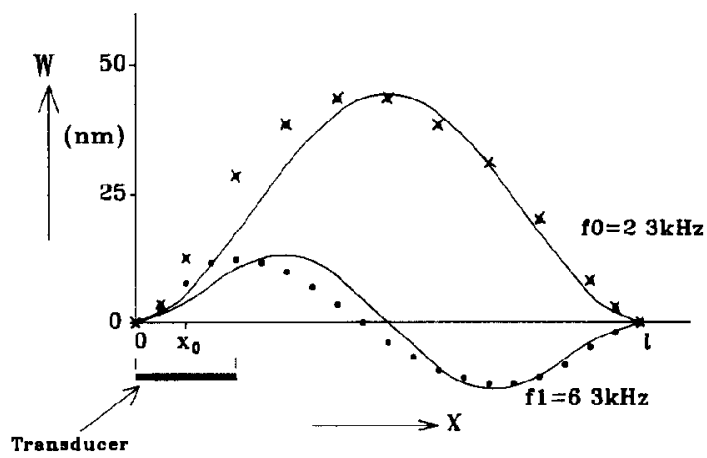

Fig 4 Calculated and measured shapes of the beam vibration $W(x)$ at the first- and second-order resonance frequencies The transducer is at $x_{\mathrm{o}} / l=01$ with $\delta / l=02$ 
$f_{\mathrm{o}}=25 \mathrm{kHz}$ The measured relation of the first two resonance frequencies $f 1 / f 0=276$ indicates that the beams are stress-free $(\sigma \rightarrow 0)$ for $P_{\text {stat }} \rightarrow 0$ With increasing power $P_{\text {stat }}$ we see that the measured resonance frequencies diverge from the expected ones when power $\boldsymbol{P}_{\text {stat }}$ approaches the critical power $P_{\text {stater }}$ This is probably caused by the clearly visible static deflection of the beam before the actual buckling load is reached This indicates that the measured resonance frequency behaviour is influenced by (Initial) beam imperfections A similar effect is found for resonatıng membranes [3] and thin-film microbridges [2]

The average temperature elevation $\Delta T_{\mathrm{av}}\left(x_{\mathrm{o}} / l\right) /$ $P_{\text {stat }}$ is recalculated from the relative frequency change $\mathrm{d} f / \mathrm{d} P_{\text {stat }}$ for $P_{\text {stat }} \rightarrow 0$ (see Figs 5 and 6) and agrees well with the analytical thermal model, which predicts that the beams have an average temperature elevation of $\Delta T_{\mathrm{av}}=118^{\circ} \mathrm{C}$ at the critical buckling load The mechanical vibration amplitude $W$ was shown to be proportional

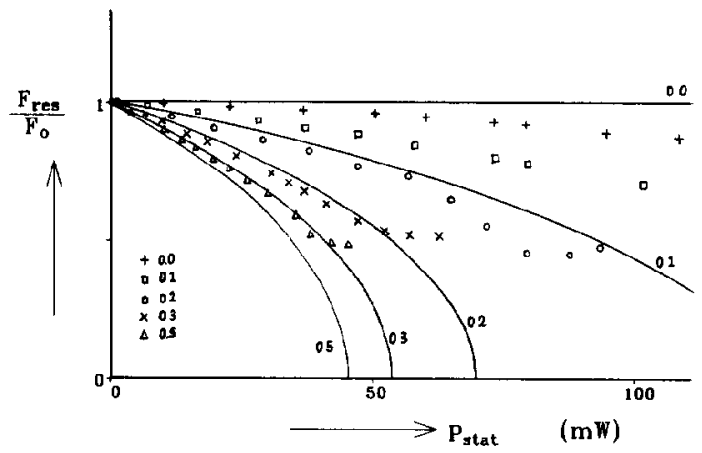

Fig 5 Calculated (using eqn (5)) and measured normalized frequency shifts as a function of the heating power $\boldsymbol{P}_{\text {stat }}$ The parameter is the relative position of the thin-film transducer $x_{\mathrm{o}} / l$

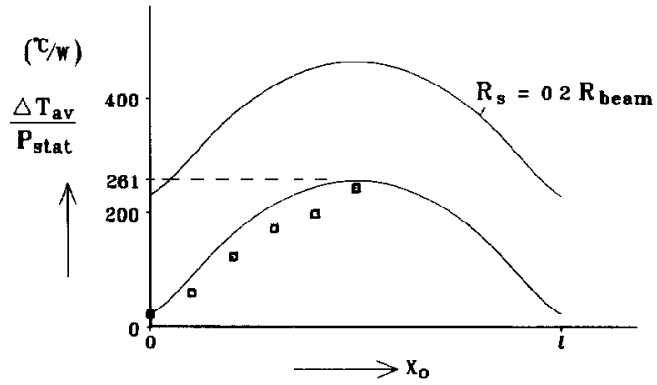

Fig 6 Calculated and measured average temperature elevations $\Delta T_{\text {av }}$ of the beam as a function of the relative transducer position $x_{\mathrm{o}} / l$ The dotted line gives $\Delta T_{\mathrm{av}}$ for an extra thermal impedance $R$, between the beam ends and the surroundings

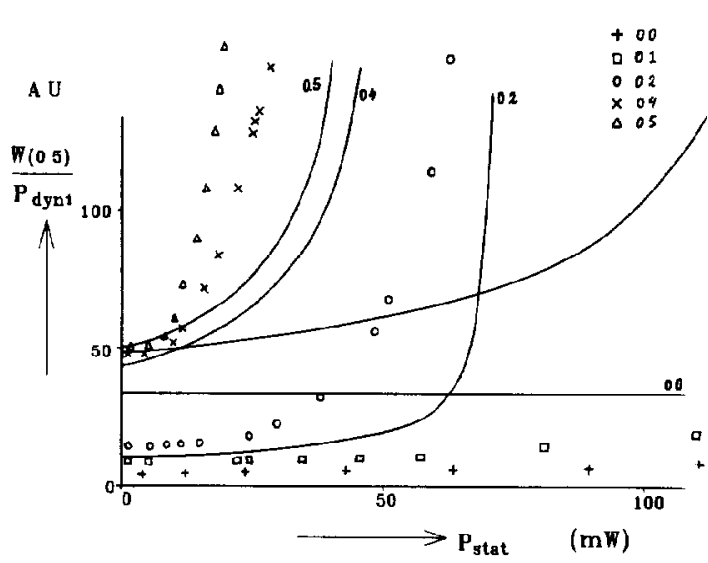

Fig 7 The vibration amplitudes relative to $P_{\text {dynl }}$ as a function of the static power $P_{\text {stat }}$ The lines show the expected behaviour assuming constant vibration energy The parameter is the relative transducer position $x_{0} / l$

to the dynamic power $P_{\text {dyn }}$ only if the static power $P_{\text {stat }}$ was kept constant $O$ n the contrary, when the static power $P_{\text {stat }}$ is vaned, the expected vibration amplitude increases according to $W \sim 1 /\left(1-P_{\text {stat }} / P_{\text {stater }}\right)^{1 / 2}$, assuming a decreasing frequency according to eqn (2) and a constant vibration energy The measured stronger increase for $W$ as illustrated in Fig 7 , may be caused by the frequency dependence of the driving moment $M_{\mathrm{d}}$ or by the static deflection also induced by $P_{\text {stat }}$ The vibration amplitude $W$ as a function of the relative transducer position $x_{0} / l$ shows a poor resemblance to the theory, as shown in Fig 8 Possible causes for the differences are (1) the different quality factors of the vanous beams, (11) excitation of the beam due to the dynamic elongation under the resistor (another excitation mechanism), and (111) the vibration amplitude depends on a static beam deflection (for $P_{\text {stat }}=0$ due to the realization process)

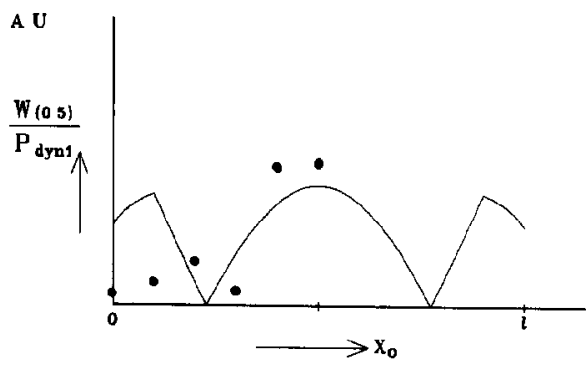

Fig 8 Calculated and measured vibration amplitudes as a function of the relative transducer position $x_{\mathrm{o}} / /$ (measured for $P_{\text {stat }} \rightarrow 0$, see $F_{1 g}$ 7) 


\section{Conclusıons}

Thermal excitation inherently causes a nse in temperature of the resonator This temperature rise induces an axial stress which significantly reduces the resonance frequency This influence is much larger than the influence of the temperature dependency of the material parameters $\rho$ and $E$ The influence of the transducer position $x_{\mathrm{o}} / l$ on the average temperature elevation $\Delta T_{\mathrm{av}}$ and the critical buckling power $P_{\text {statcr }}$ is quantitatively described and shows a good agreement with experimental results The extreme sensitivity to stresses indicates that the thermal as well as the mechanical packaging of resonators may have a significant influence on the performance The beam mechanics in the vicinity of the buckling load and the effectiveness of the excitation mechanism as a function of the axial stress $\sigma$ need investigating further

\section{Acknowledgement}

The authors would like to thank Gerard Roelofs from the Sensors and Actuators Research Unit laboratory for his help realızing the test samples

\section{References}

1 R M Langdon, Resonator sensors - a review, $\boldsymbol{J}$ Phys $E$ Sct Instrum, 18 (1985) 103-115

2 S Bouwstra, R Legtenberg, $H$ A $C$ Tilmans and $M$ Elwenspoek, Resonating microbndge mass flow sensor, Sensors and Actuators, A2I-A23 (1990) 332-335

$3 \mathrm{~S}$ Bouwstra, $\mathbf{P}$ Kemma and $\mathbf{R}$ Legtenberg, Thermally excited resonatung membrane mass flow sensor, Sensors and Actuators, 20 (1989) 213-223

4 M B Othman and A Brunnschweller, Electrothermally excited silicon beam mechanical resonators, Electron Lett, 23 (1987) 728-730

$5 \mathrm{M}$ V Andres, $\mathrm{K}$ W H Foulds and $\mathrm{M} J$ Tudor, Sensitıvity and mode spectrum of a frequency-output silicon pressure sensor, Sensors and Actuators, 15 (1988) 417-426

$6 \mathrm{~T}$ S J Lammernk and W Wlodarsk1, Integrated thermally excited resonant diaphragm pressure sensor, Proc Int Conf Soldd-State Sensors and Actuators (Transducers '85), Philadelphia, PA, US A, June 11-14, 1985, pp 97100

7 K E B Thornton, D Uttamchandan and B Culshaw, Temperature dependence of resonance frequency in optscally excited diaphragms, Electron Lett, 22 (1986) 12311234

$8 \mathrm{R}$ J Wilfinger, P H Bardell and D S Chhabra, The resonistor a frequency selective device utilizang the mechanIcal resonance of a slicon substrate, IBM $J$ Res Develop, 12 (1968) 113-118

$9 \mathrm{~J} \mathrm{M}$ Gere and S P Timoshenko, Mechanics of Materials, Van Nostrand Reinhold, New York, 2nd edn, 1988, p 564

10 T S J Lammerink and $M$ Elwenspoek, Dynamic thermal moment for thermal excitation of resonator, to be published 